\title{
Hybrid Structures of NiTi and Carbon Nanotubes
}

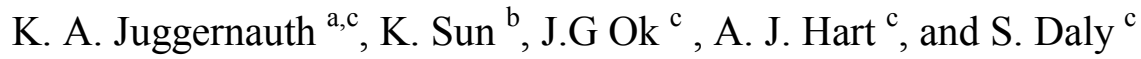

${ }^{a}$ Macromolecular Science and Research Center, University of Michigan, 2455 Hayward St, Ann Arbor, MI 48105

${ }^{b}$ Electron Microbeam Analysis Laboratory, Department of Materials Science and Engineering, University of Michigan, 2455 Hayward St, Ann Arbor, MI 48105

${ }^{\mathrm{c}}$ Department of Mechanical Engineering, University of Michigan, 2350 Hayward St, Ann Arbor, MI 48109

Hybrid materials combining the attractive properties of multiple types of nanostructures are useful for applications including energy storage and biosensing; and their synthesis enables new understanding and control of material properties that are governed by atomic interfaces [1]. Carbon nanotubes (CNTs) have generated tremendous interest due to their unique electrical, optical and mechanical properties [2]. Vertically aligned CNT "forests" grown by catalyst mediated chemical vapor deposition (CVD) can serve as a template for the subsequent deposition of a second material, creating a hybrid 3-D structure [3]. Our goal is to combine the biocompatible shape memory alloy Nitinol (NiTi) with CNTs, and evaluate the utility of this novel 3-D hybrid structure for biomedical and micromechanical devices. The properties of NiTi are determined by a diffusionless solid state phase transformation caused by a change in the crystal structure. NiTi thin films are attractive due to high energy density and fast response relative to the bulk material [4]. This paper reports the synthesis and characterization of NiTi/CNT hybrid structures.

Vertically aligned forests of multi-walled CNTs were grown by CVD [5]. A 500nm thick NiTi film $(53.5 \mathrm{wt} \% \mathrm{Ni})$ was subsequently sputtered onto the CNT forests. After deposition, the samples were annealed in a flowing $\mathrm{He}$ atmosphere at $450^{\circ} \mathrm{C}$ for $60 \mathrm{~min}$.

SEM imaging (Fig 1) shows that the NiTi film is continuous and relatively smooth on $\mathrm{Si}$, and has columnar morphology on the top surface of a CNT forest. XRD conducted before and after annealing shows sharp peaks between $2 \theta=42-45^{\circ}$, indicating a significant improvement in the sample crystallinity for both $\mathrm{NiTi} / \mathrm{Si}$ and NiTi/CNT. HRTEM and SAED pattern of a single column extracted from the NiTi/CNT indicates that it is highly polycrystalline with random orientation. EDS of the column confirms that it comprises $\mathrm{Ni}$ and $\mathrm{Ti}$. We have also identified metal nanocrystals on the sidewalls of conformally coated CNT bundles. Kinking and buckling of the CNT walls at the $\mathrm{NiTi} / \mathrm{CNT}$ interface was also observed. Upon annealing, further crystallization was confirmed by well defined XRD peaks for both NiTi/Si and NiTi/CNT. In comparison, the annealed NiTi/CNT samples exhibit well-defined diffraction spots, confirming an increase in crystallinity. We are performing additional structural and mechanical characterization of the NiTi/CNT hybrids, seeking to determine how nanoscale interfaces and polycrystalline order may affect shape memory behavior.

\section{References}

[1] D. R. Rolison et al., Chemical Society Reviews (2009) 38, 226

[2] M. S. Dresselhaus et al. Carbon Nanotubes: Synthesis, Structure, Properties, and Applications, Springer, 2001 
[3] W. Lu and C. M. Lieber, Journal of Physics D-Applied Physics (2006), 39, R387

[4] K.S.S. Eswar Raju, et al., Mater. Sci. Eng. A 476 (2008), 267-273

[5] A.L Hart and A.H.Slocum J. Physical Chemistry B 110(16):8250-8257, 2006.

[6] The authors would like to thank Prof D.S.Grummon for deposition of the NiTi thin films.
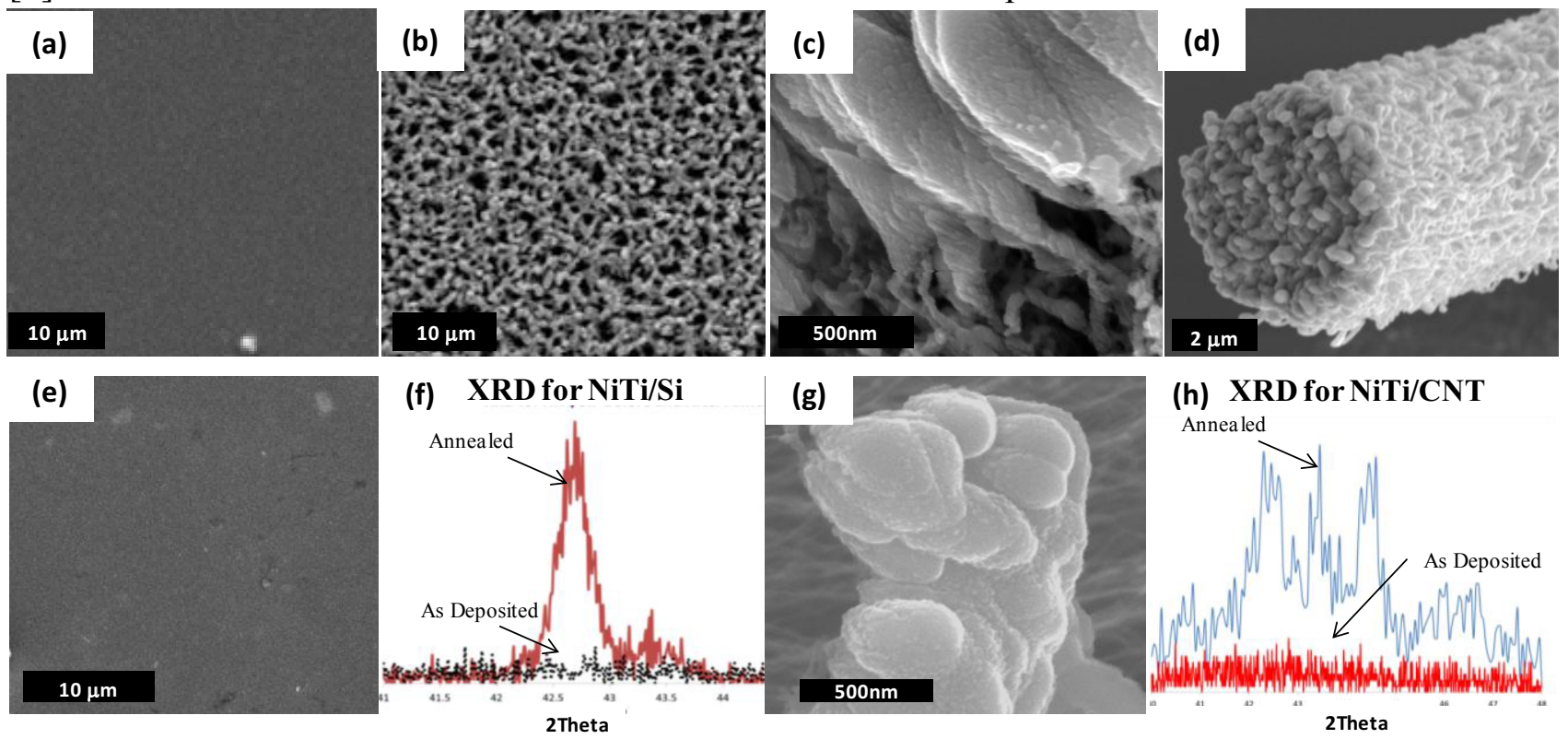

FIG. 1: Top surface morphology of as-deposited NiTi film on (a) Si (b) CNT, (c) NiTi/CNT interface; (d) Conformally coated pillar; SEM and XRD of annealed (e, f) NiTi/Si (g, h) NiTi/CNT
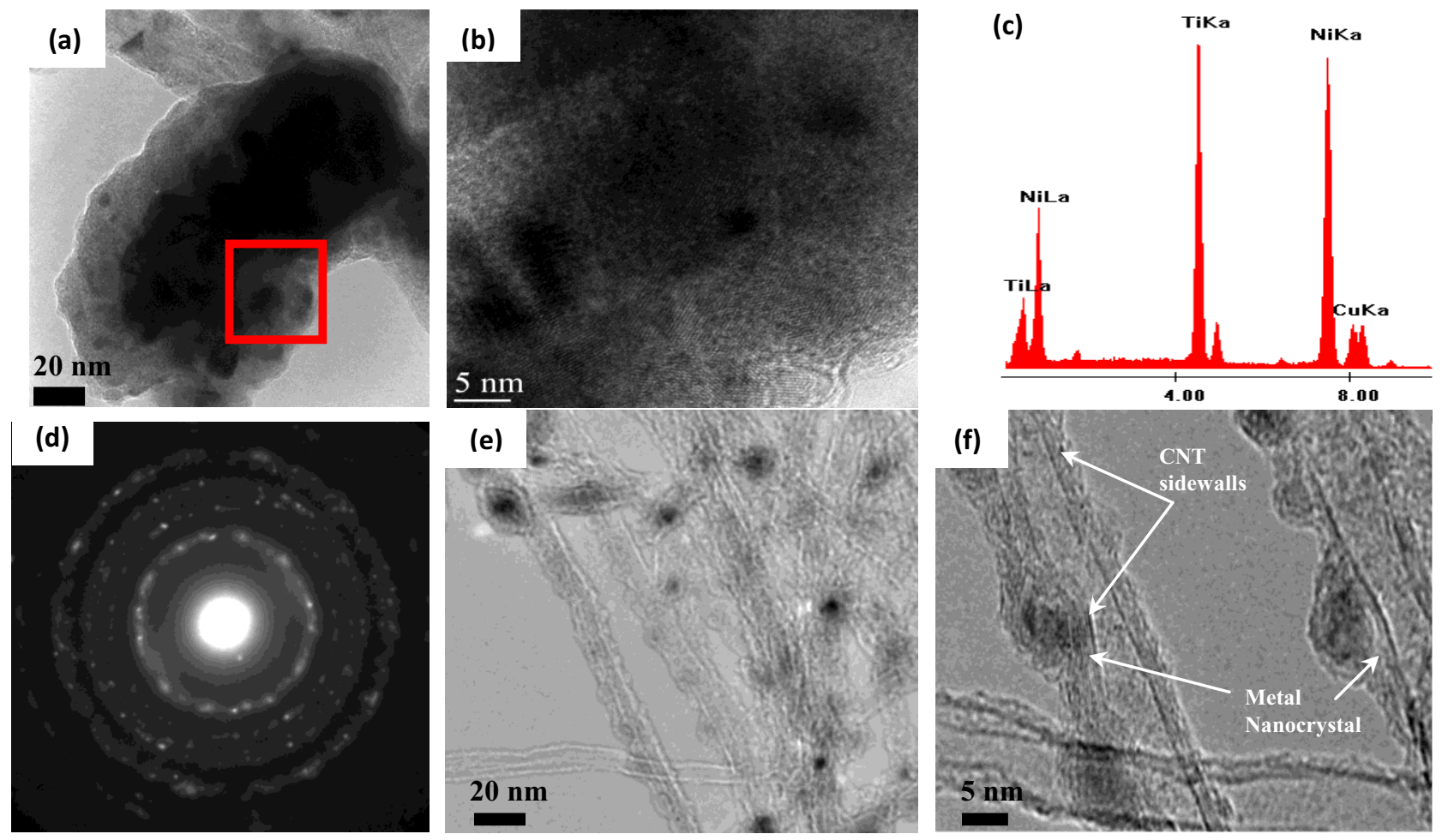

FIG. 2. Characterizaton of NiTi/CNT "column" extracted from forest (a,b) TEM images, (c) EDS, (d) SAED; (e) TEM images of NiTi on CNTs showing (e) conformal coating, and (f) isolated metal nanocrystals. 